\title{
HULC and Linc00152 Act as Novel Biomarkers in Predicting Diagnosis of Hepatocellular Carcinoma
}

\author{
Jun Lia,b Xiaochen Wang ${ }^{\mathrm{a}} \quad$ Junwei Tang ${ }^{\mathrm{a}}$ Runqiu Jiang ${ }^{\mathrm{a}}$ Wenjie Zhang ${ }^{\mathrm{a}} \quad$ Jie Ji $^{\mathrm{a}}$ \\ Beicheng Sun ${ }^{\mathrm{a}}$ \\ aiver Transplantation Center of the First Affiliated Hospital and State Key Laboratory of Reproductive \\ Medicine, Nanjing Medical University, Nanjing, 'Department of General Surgery, The Affiliated \\ Jiangning Hospital of Nanjing Medical University, Nanjing, P.R. China
}

\section{Key Words}

Hepatocellular carcinoma • Long non-coding RNAs • HULC • Linc00152 • Biomarker

\begin{abstract}
Background/Aims: The alterations of long non-coding RNAs (IncRNAs) are related to multiple diseases. They can be detected in plasma as biomarkers for the diagnosis of multiple diseases. In this study, we aimed to determine the expression of circulating IncRNAs in human, which may be promising biomarkers for the diagnosis of hepatocellular carcinoma (HCC). Methods: Eight IncRNAs were chosen as candidates on the basis of the literature to evaluate the diagnostic value and accuracy of the plasma IncRNA profiling system. The candidate IncRNAs were validated by qRT-PCR arranged in the training and validation sets. Additional double-blind testing was performed in 20 patients clinically suspected of having HCC. Results: Circulating HULC and Linc00152 were significantly up-regulated in plasma samples of HCC patients during training set and validation set. Areas under the receiver operating characteristic (ROC) curves of the validated two IncRNAs signature were 0.78 and 0.85 , respectively. Combination of HULC and Linc00152 possessed a moderate ability to discrimination between $\mathrm{HCC}$ and control with an area under ROC value of 0.87 while the combination of AFP was 0.89 with a positive correlation with tissues expression. Conclusions: Our results suggest that both plasma levels of HULC and Linc00152 achieve a fine diagnostic accuracy in diagnosing ontogenesis and metastasis of HCC and may act as novel biomarkers for HCC.
\end{abstract}

Copyright $(2015$ S. Karger AG, Basel

\section{Introduction}

Hepatocellular carcinoma (HCC) is the fifth most commonly diagnosed cancer in men and seventh in women around the world, and the mortality of HCC

J. Li, X. Wang and J. Tang contributed equally to this work.

Dr. Beicheng Sun

KARGER 125
Liver transplantation Center, The First Affiliated Hospital of Nanjing Medical University, 300 Guangzhou Road, Nanjing, Jiangsu Province, (P.R. China)

Tel. +86 25 68136746, Fax +86 25 86560946, E-Mail sunbc@njmu.edu.cn 
ranks as a second in former and as a sixth in latter. The half of new cases of death occurs in China every year [1-3]. The prognosis of patients with HCC is poor and once HCC invades other organs and tissues or spreads distantly, the clinical outcome may be even worse [45]. Therefore, early detection of HCC is of great importance. The diagnosis of HCC mainly depends on imaging techniques, which are less efficient for detecting early HCC [6]. Plasma tumor marker detection is an ideal method for screening patients at an early stage and prefiguring their prognosis. At present, plasma alpha fetoprotein (AFP) is the most widely used tumor marker for HCC, however, AFP is limited by its low sensitivity and specificity [7]. Thus, looking for some new tumor markers with high sensitivity and specificity to detect the tumorigenesis and recurrence of HCC independent or as a supplement of AFP is badly needed. Cell-free nucleic acids (cfNA), such as DNA, mRNA and microRNA can be detected in the plasma of patients with cancer. A number of studies have implicated that changes in the levels of cfNA was linked to tumorigenesis and progression [8,9].

Long non-coding RNAs (lncRNAs) are one type of non-protein coding transcripts with more than 200 nucleotides in length. LncRNAs are important regulatory molecules at every level of cellular physiology, alterations of which are related to multiple diseases, including cancers [10]. Some lncRNAs can be detected in plasma of both healthy people and certain patients. LncRNAs have been reported as biomarkers for the diagnosis of multiple diseases $[11,12]$, including cancers, such as cancers of colorectum, prostate and bladder [13-15]. Some previous studies showed that many lncRNAs were up-regulated or down-regulated significantly in tumor tissues compared with non-tumor tissues [16-20]. However, the aberrant expression of these lncRNAs in HCC has not been clarified yet. Although several other studies showed that some IncRNAs were up-regulated or down-regulated in HCC [2123], their expression in plasma of HCC patients has not been investigated.

In the present study, we chose eight IncRNAs (UCA1, TUG1, CCAT1, MEG3, Linc00152, HULC, MALAT1 and GAS5) as plasma biomarker candidates on the basis of the literature. Relative expression levels of these candidates in plasma samples were analyzed in a small cohort of HCC patients and healthy controls, 32 patients with chronic hepatitis were regarded as disease controls. To identify a novel biomarker in predicting the diagnosis of HCC, a case-control study was designed to validate the significantly different relative expression of potential biomarkers in plasma samples of a large cohort of HCC patients and healthy controls.

\section{Materials and Methods}

Study design

Discovery phase. For the purpose of indentifying plasma lncRNAs as potential biomarkers, we searched candidate databases of IncRNAdb, LNCipedia, and LncRNA Disease. Previous studies showed that UCA1, TUG1, CCAT1 and Linc00152 were up-regulated obviously in tumor tissues compared with nontumor tissues [16-19], while MEG3 was down-regulated significantly in tumor tissues. Regulations of all these IncRNAs in HCC have not been clarified yet. Several other studies have demonstrated that HULC and MALAT1 were overexpressed in HCC, while GAS5 was down-regulated in HCC [21, 22]. Nevertheless, the expression of these IncRNAs in plasma of HCC patients has not been performed. Thus, we selected eight IncRNAs (UCA1, TUG1, CCAT1, MEG3, Linc00152, HULC, MALAT1 and GAS5) as candidates for this research.

Training set. All candidates were tested in an independent cohort of 48 plasma samples from 24 HCC patients who underwent hepatic resections at the First Affiliated Hospital of Nanjing Medical University and 24 healthy controls. The expression levels of these candidates were analyzed in all plasma samples, and comparative delta-Ct method algorithms were used to analyze the difference between HCC patients and health controls. We found the expression of three lncRNAs among the eight candidates significantly differed between the 2 groups. Then, we selected these IncRNAs as potential biomarker candidates for further investigation.

Validation set. A case-control study was designed to validate the obvious difference of relative expression levels of the selected potential biomarkers candidates in another independent cohort of plasma 


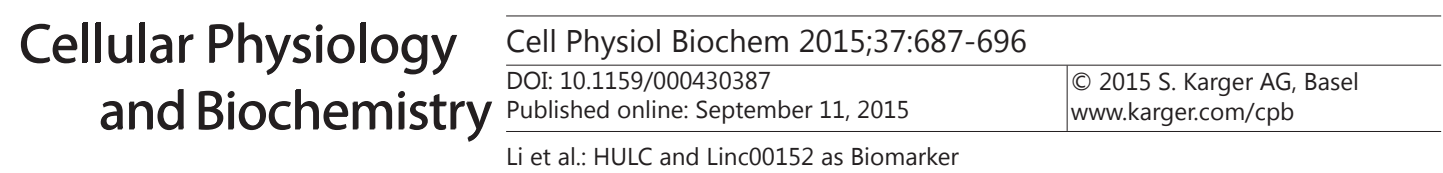

samples from 66 HCC patients who underwent hepatic resections at the First Affiliated Hospital of Nanjing Medical University and 53 healthy controls. We found two IncRNAs of the three candidates were up-regulated significantly in HCC patients. Then, we analyzed the relationship between the two IncRNAs expression and the clinicopathological features of HCC patients, including age, gender, tumor size, differentiation grade, tumor number, tumor capsular, TNM stage and metastasis. Finally, we used receiver operating characteristic (ROC) curve to examine the diagnosis value of these two IncRNAs. The overflow was presented in Fig. 1.

\section{Patient samples and clinical specimens}

Study data were obtained from patients with HCC who underwent hepatic resections between August 2011 and September 2013 at Liver Transplantation Center of the First Affiliated Hospital, Nanjing Medical University (Nanjing, PR China). Controls were from healthy volunteers without any health problems during their health check-ups at the First Affiliated Hospital of Nanjing Medical University. The research protocol was approved by the Institutional Ethics Committee of the First Affiliated Hospital of Nanjing Medical

Fig. 1. Overview of the workflow.

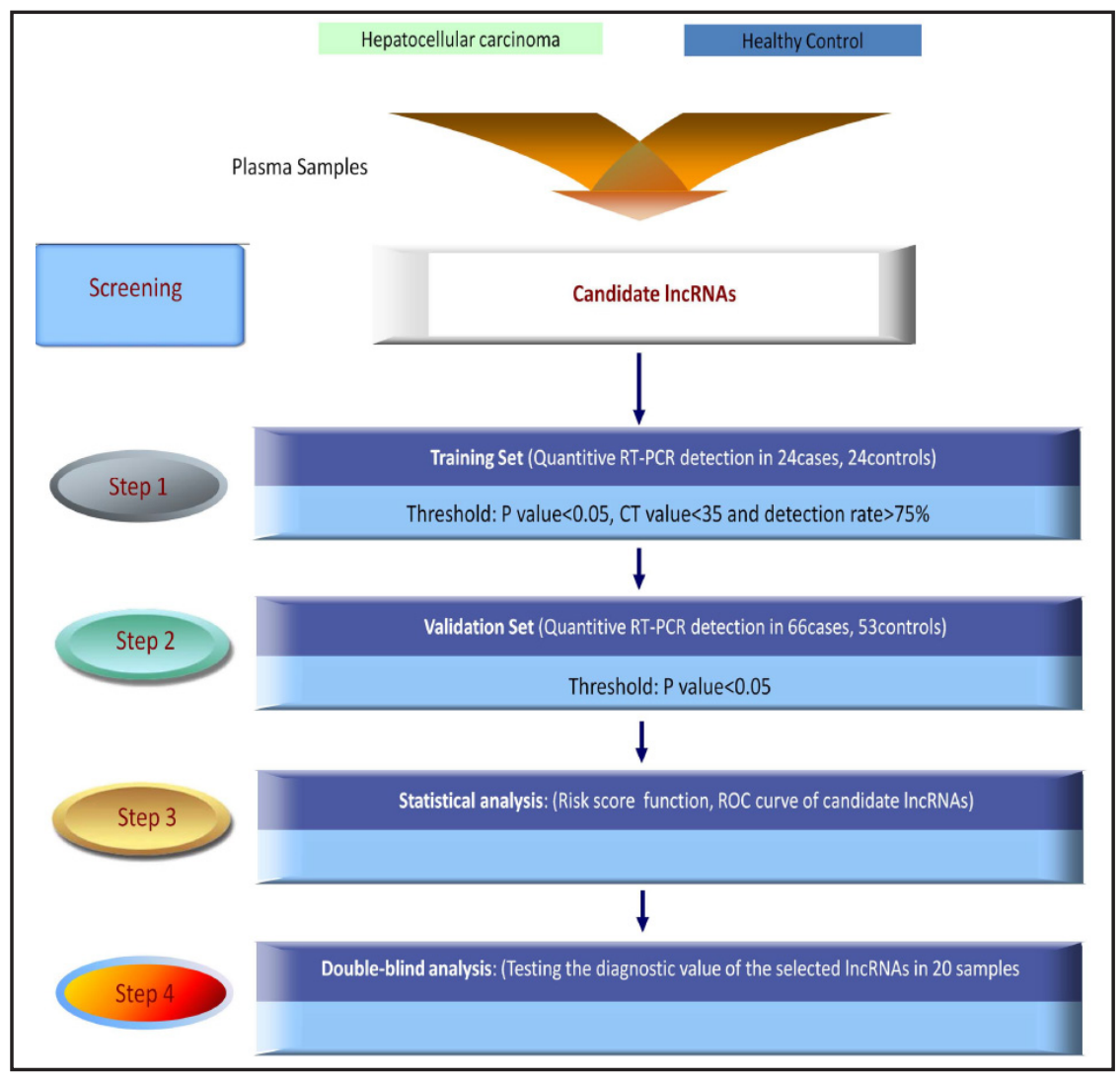

Table 1. The primer of eight lncRNAs candidates and 5S rRNA

\begin{tabular}{lll}
\hline & Forward primer $\left(5^{\prime}-3^{\prime}\right)$ & Reverse primer $\left(5^{\prime}-3^{\prime}\right)$ \\
\hline UCA1 & CTCTCCATTGGGTCACCATTC & GCGGCAGGTCTTAAGAGATGAG \\
TUG1 & TAGCAGTTCCCAATCCTTG & CACAAATTCCATCATTCCC \\
CCAT1 & CATTGGGAAAGGTGCCGAGA & ACGCTTAGCCATACAGAGCC \\
MEG3 & GCCAAGCTTCTTGAAA GGCC & TTCCACGGAGTAGAGCGAGTC \\
Linc00152 & CTCCAGCACCTCTACCTGTTG & GGACAAGGGATTAAGACACACA \\
HULC & ATCTGCAAGCCAGGAAGAGTC & CTTGCTTGATGCTTTGGTCTGT \\
MALAT1 & AAAGCAAGGTCTCCCCACAAG & GGTCTGTGCTAGATCAAAAGGCA \\
GAS5 & GGAGAGGGAGCCTGAGAAACG & TTACAGGGCCTCGAAAGAGTCC \\
\hline
\end{tabular}




\section{Cellular Physiology Cell Physiol Biochem 2015;37:687-696 \\ \begin{tabular}{l|l|l} 
DOI: 10.1159/000430387 & (C) 2015 S. Karger AG, Basel
\end{tabular} \\ Li et al.: HULC and Linc00152 as Biomarker}

University. Written informed consent was obtained from every participant. Peripheral blood samples and tissues samples of patients were collected before surgery. Blood samples were collected in a separate vacuum cube, followed by centrifugation at 3,000 rpm for $10 \mathrm{~min}$. All samples were stored at $-80^{\circ} \mathrm{C}$ until further analysis. The relevant clinical data of all patients were available. All patients were diagnosed by histological examination.

Quantitative real-time PCR ( $q R T$-PCR)

Total RNAs were obtained from plasma samples using TRIzol reagent as described by the manufacturer (Invitrogen Life Technologies Co, Carlsbad, CA, USA). For mRNA detection, total RNAs (500ng) were reverse transcribed using the reverse transcription kit (Takara, Tokyo, Japan). The quality of RNA samples was assessed by a UV spectrophotometer (Bio- Rad, Hercules, CA, USA), and the 260/280nm absorbance ratio of samples were limited to 1.8-2.0. qRT-PCR was performed in 384-well plates by use of an ABI Prism 7900HT (Applied Biosystems, Foster City, CA, USA) instrument according to the manufacturer's instructions. The qPCR reaction mixture consisted of $2.5 \mu \mathrm{l}$ of SYBR® Premix Ex Taq ${ }^{\mathrm{TM}}$ (Perfect Real Time) (2×) (Takara: DRR081A TaKaRa, Japan), $0.5 \mu \mathrm{l}$ of primers, $0.1 \mu \mathrm{l}$ of ROX Reference Dye (50×),1 $\mu \mathrm{l}$ of the cDNA solution and nuclease-free $\mathrm{H}_{2} \mathrm{O}$ at a final volume of $5 \mu \mathrm{l}$. 5S rRNA was used as an internal control while the cel-miR-39 was conducted as the external control. The primer of eight lncRNAs candidates and internal control were presented in Table 1. All samples were performed for four times. A melt-curve analysis was carried out at the end of the amplification to verify the non-specific amplification.

\section{Statistical analysis}

The results of qRT-PCR and other variables were presented as mean (S.E.M.).The Student's t-test and Mann-Whitney unpaired test analysis of variance were used to evaluate statistical differences between HCC patients and controls. Analysis of area under the ROC curve (AUC) was used to estimate the effectiveness of lncRNAs for prediction. Statistical analysis was performed using STATA 9.2 and GraphPad prism software. In all cases, $\mathrm{P}<0.05$ was considered to be significant. All $P$ values were two-sided.

\section{Results}

Expression of eight IncRNAs candidates in HCC in Training phase

As presented in Fig. 2 and Table 2, high expression levels of HULC and Linc00152 were observed in HCC patients compared with controls, while low expression level of UCA1 in HCC patients was identified. No statistical significance was demonstrated for the expression levels of TUG1, CCAT1 MEG3, MALAT1 and GAS5 in patients with HCC in contrast to controls.

Expression of selected IncRNAs candidates for HCC in validation phase

In validation phases, our results indicated that the relative expression levels of HULC and Linc00152 were up-regulated in HCC patients compared with controls. However, the

Table 2. The relative expressions of eight lncRNAs candidates for HCC comparing control in Training phase. *indicates statistically significant

\begin{tabular}{lccc}
\hline & HCC & control & $p$ value \\
\hline UCA1 & $0.02043 \pm 0.004120$ & $0.04351 \pm 0.008908$ & $0.0239 *$ \\
TUG1 & $0.003672 \pm 0.0008270$ & $0.01192 \pm 0.006560$ & 0.3323 \\
CCAT1 & $0.6556 \pm 0.2022$ & $0.6857 \pm 0.1943$ & 0.9152 \\
MEG3 & $0.003363 \pm 0.0009886$ & $0.001408 \pm 0.0002988$ & 0.0997 \\
Linc00152 & $0.8670 \pm 0.2069$ & $0.002608 \pm 0.001010$ & $<0.0001^{*}$ \\
HULC & $0.9695 \pm 0.1615$ & $0.003385 \pm 0.0006782$ & $<0.0001^{*}$ \\
MALAT1 & $0.003637 \pm 0.0008856$ & $0.005556 \pm 0.001307$ & 0.2239 \\
GAS5 & $0.006502 \pm 0.001176$ & $0.004210 \pm 0.0007896$ & 0.1021 \\
\hline
\end{tabular}




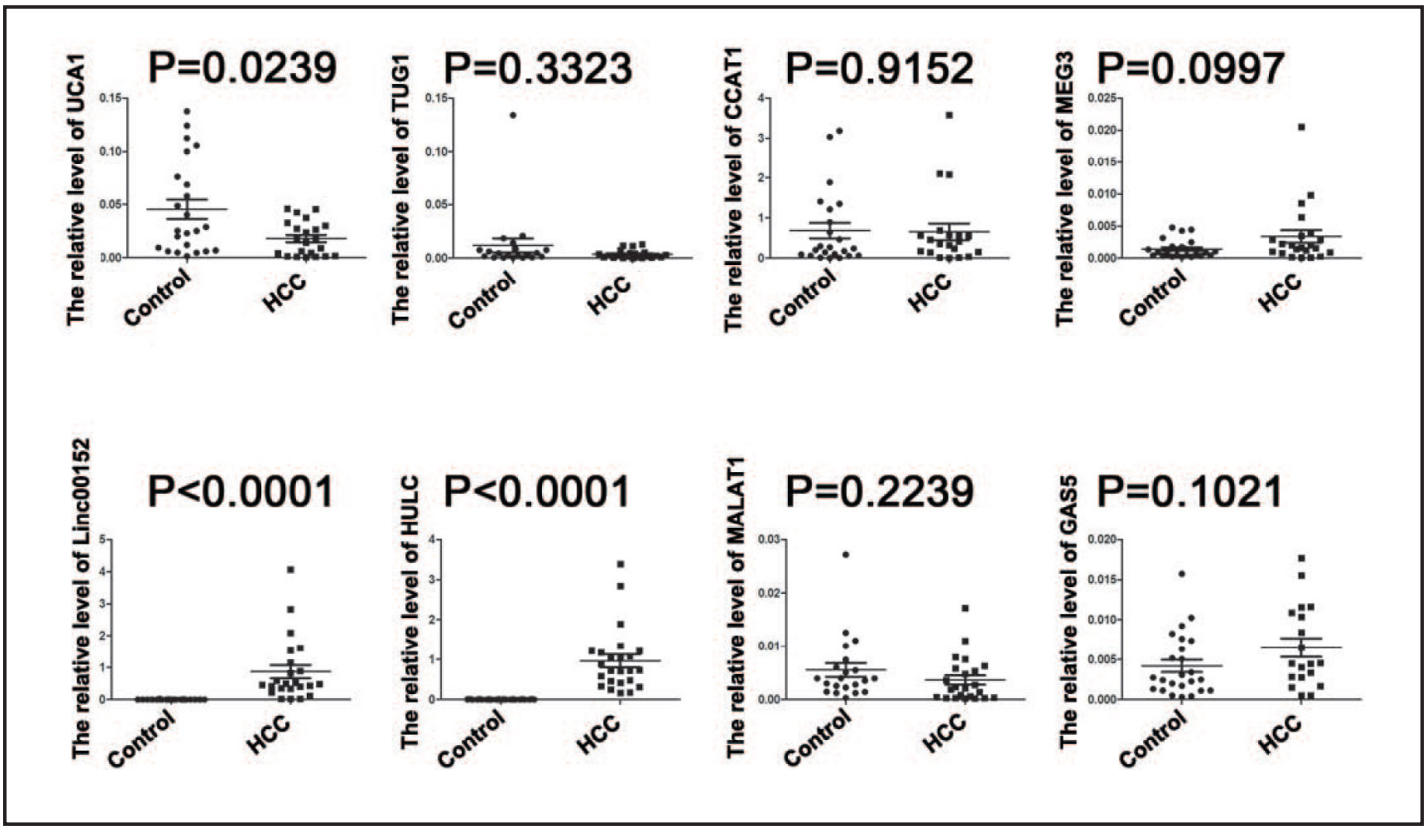

Fig. 2. HULC, Linc00152 and UCA1 were confirmed as the candidate lncRNAs in training set analysis. Increased level of HULC, Linc00152 and UCA1 were confirmed by RT-PCR in groups. Data were presented as plot of the median and range of log-transformed relative expression level.

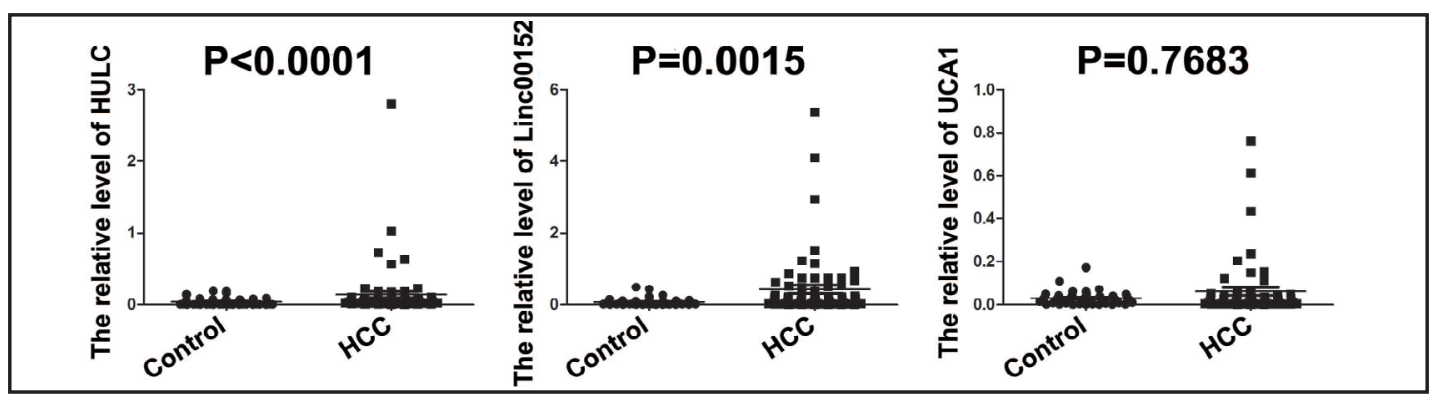

Fig. 3. HULC and Linc00152 were confirmed the up-regulation in validation set. Increased level of HULC and Linc00152 were confirmed by RT-PCR in groups. Data were presented as plot of the median and range of log-transformed relative expression level.

expression level of UCA1 in HCC patients showed no statistical significance when comparing with controls (Fig. 3).

\section{Clinicopathological data analysis of HCC patients}

The median value was set as the cut off value to classify the expression levels of HULC and Linc00152. As presented in Table 3, all clinicopathological data were divided into positive and negative groups according to different grouping criteria and were analyzed by chi-squared test or Fisher exact test. Tumor size, differentiation grade, TNM stage were grouped by 5 $\mathrm{cm}$ (the threshold of indicating small HCC), edmondson-Steiner grading, TNM Classification of Malignant Tumors (7th edition) as well as the current drug treatments(including HBC Antiviral therapy and Chemotherapy) and underlying disease ( $\mathrm{CH}$ or other disease) was calculated. The clinicopathological relevance analysis of HCC patients demonstrated that the HULC expression significantly correlated with tumor size and tumor capsular, while the expression of Linc00152 was significantly related to differentiation grade, tumor size, TNM stage and tumor capsular.

\section{KARGER}


Table 3. The clinicopathological relevance analysis of HULC and Linc00152 relative expression levels in HCC patients. Clinicopathological data of 66 HCC patients were analyzed. For the relative expression of HULC and Linc00152, the median expression level was used as the cut off value. Data were analyzed by chi-squared test. *indicates statistically significant

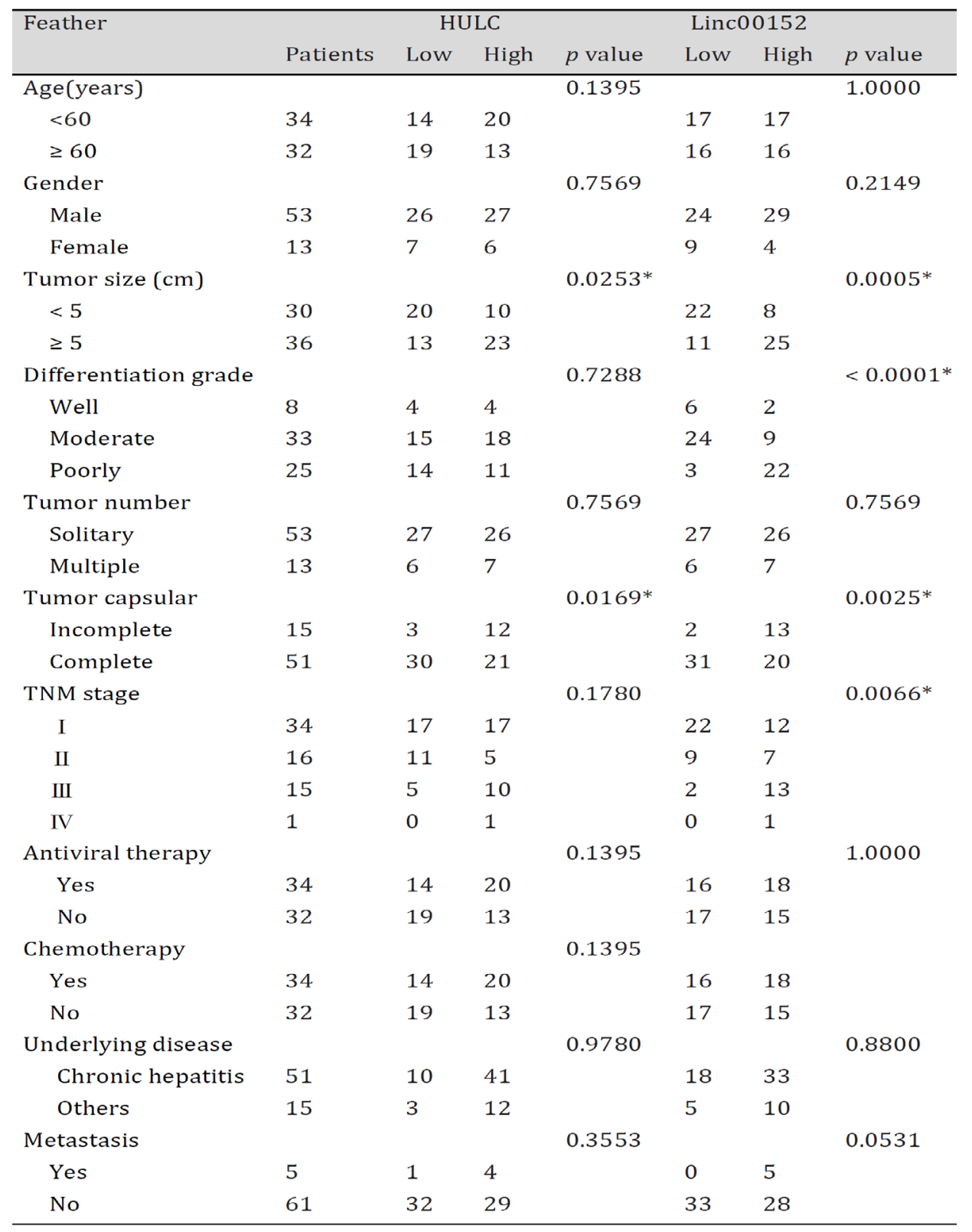

Receiver operating characteristic (ROC) curve analysis for predicting the diagnosis of HCC

ROC curve analysis was used to evaluate the predicting diagnosis value of lncRNAs for HCC. Areas under the ROC curves of the validated two IncRNAs signature were 0.78 and 0.85 , respectively. Combination of HULC and Linc00152 possessed a moderate ability for discrimination between HCC patients and controls with an area under the ROC curve of 0.87 , while the area was equal to 0.89 when they combine with AFP (Fig. 4).

Double-blind test

Another 20 plasma samples were tested in a double-blind fashion to validate the predictive ability of the two IncRNAs. We used the same risk score formula to analyze the 
Fig. 4. ROC analysis of the two potential biomarkers for HCC as well as the combination of AFP.

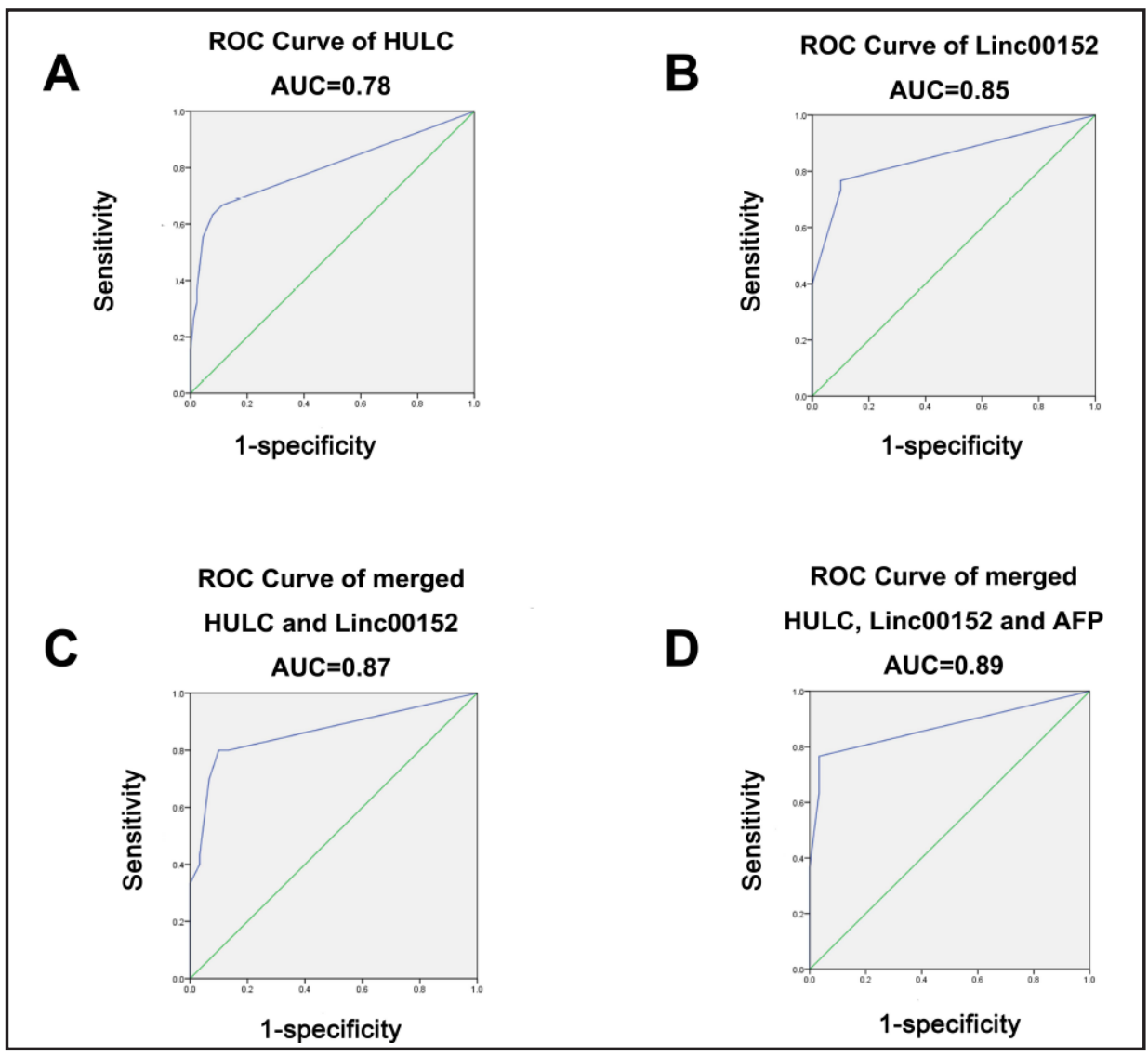

Fig. 5. Tissues correlated HULC and Linc00152 up-regulated specific in plasma of HCC. (A) Pearson correlation analysis of HULC and Linc00152 expression level between circulation and tissues. (B) The expression level of Linc00152 and HULC in $\mathrm{HCC}, \mathrm{CH}$ and control group. Data were presented as plot of the median and range of log-transformed relative expression level. *indicated $\mathrm{P}<0.05$.

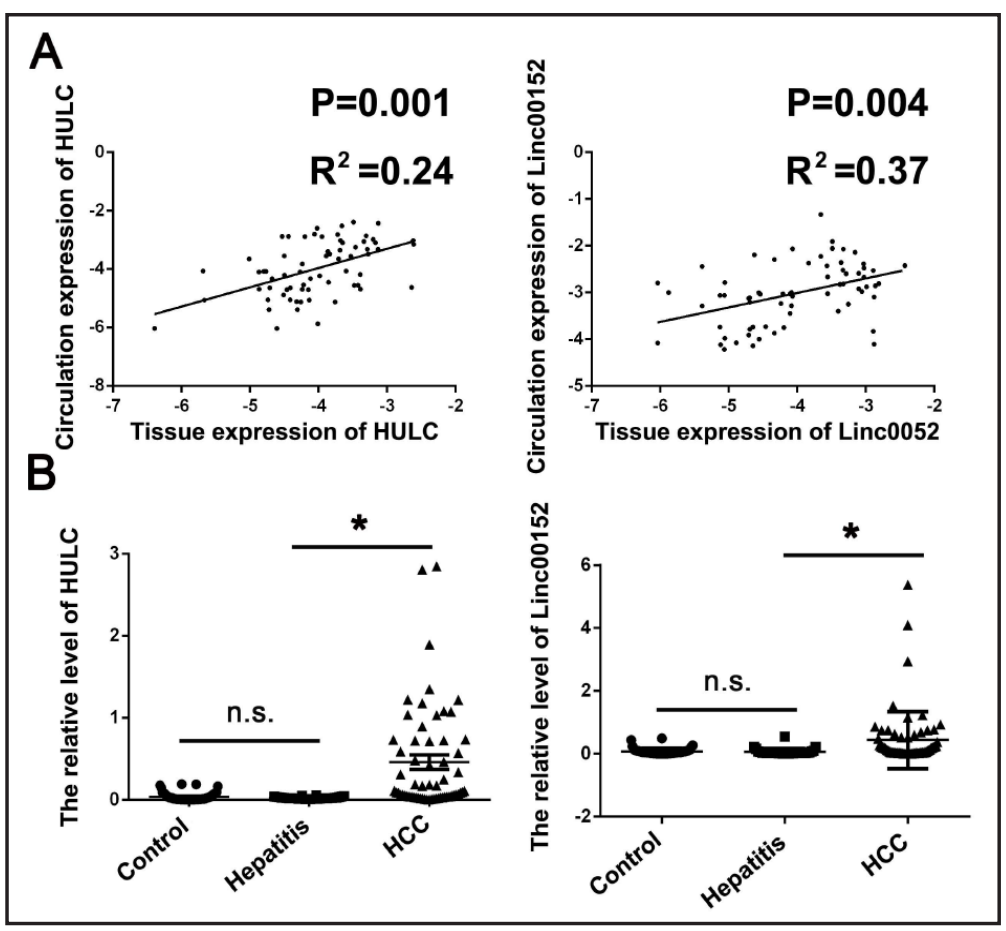

expression of the 2 IncRNAs in those plasma samples and classified them into a high-risk group and a low-risk group. On the basis of the pathologic diagnosis, the accuracy rate of the two-lncRNA profile as HCC signature was $81.9 \%$ (nine of eleven HCC cases were identified accurately). 
Fig. 6. The products of the amplification fragment were detected by agarose electrophoresis.

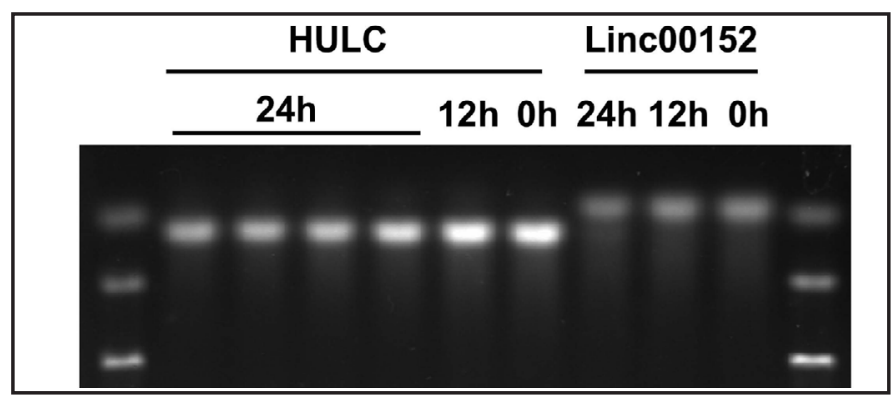

Specificity and stability detection of endogenous IncRNAs in human plasma

We further analyzed the expression of the two lncRNAs in the corresponding tissues of the same patients we included. Pearson correlation analysis showed a positive correlation of HULC and Linc00152 between their circulation expression levels and tissues expression levels (Fig. 5A). Since most patients investigated in our study were HBV positive, we enrolled another 32 patients suffering from chronic hepatitis $(\mathrm{CH})$ as disease control group. The expression levels of Linc00152 and HULC were detected in these patients. As presented in Fig. 5B, there was no significant difference for the expression of the two factors between the control group and $\mathrm{CH}$ group, while an aberrant expression was obtained when comparing with the HCC group.

We next amplified the two IncRNAs in healthy controls, and detected the product of amplification by agarose electrophoresis. The bands presented in Fig. 6 indicated that both the two lncRNAs were detectable in human plasma. We further incubated human plasma obtained from three healthy controls at room temperature for $0 \mathrm{~h}, 12 \mathrm{~h}$ and $24 \mathrm{~h}$. All of the process had minimal effects on the concentration of the two lncRNAs, demonstrating that these lncRNAs were sufficiently stable in human plasma.

\section{Discussion}

Due to the difficulty in early discovery, diagnosis and treatment, HCC is the most common malignant tumor with a higher mortality and an extremely low 5-year survival rate in the world $[1,24]$. AFP, the main plasma tumor marker for HCC diagnosis and screening, has a potential capacity to screen early stage patients but with low sensitivity [7]. Furthermore, its specificity for HCC diagnosis is also not well because AFP can often rise in the absence of HCC, such as chronic hepatitis and cirrhosis [25-27]. Therefore, we aimed to seek a newly potential tumor marker with relative higher sensitivity and specificity or as a supplement of AFP for HCC.

Many researchers have demonstrated that IncRNAs play a very important role in tumor occurrence, invasion, and metastasis and can be considered as biomarkers in many human cancers $[13-15,28]$. Some previous studies have shown that many lncRNAs were upregulated or down-regulated dramatically in tumor tissues in contrast to non-tumor tissues [16-20]. However, the regulation of these lncRNAs in HCC remains largely unknown. Several previous reports presented that some IncRNAs were overexpressed or down-regulated in HCC [21-23]. Nevertheless, due to their characteristic of unstable expression and easily degradation in plasma, the expression of these lncRNAs in plasma of HCC patients has not been investigated. Thus, we chose eight IncRNAs (UCA1, TUG1, CCAT1, MEG3, Linc00152, HULC, MALAT1 and GAS5) as plasma biomarker candidates on the basis of the literature in this study.

Firstly, we detected the levels of these plasma IncRNAs from a small cohort of HCC patients and healthy controls- by qRT-PCR. The relative expression of these lncRNAs was calculated by use of delta-Ct method algorithms. The plasma levels of HULC and Linc00152 significantly increased in HCC patients, while the plasma level of UCA1 obviously decreased in HCC patients. Then, we conducted a case-control study to validate the outcome of training phase. Levels of HULC, Linc00152 and UCA1 were detected in a large cohort of HCC patients 


\begin{tabular}{|c|c|c|}
\hline Cellular Physiology & Cell Physiol Biochem 2015;37:687-696 & \\
\hline and Biochemistry & $\begin{array}{l}\text { DOI: 10.1159/000430387 } \\
\text { Published online: September 11, } 2015\end{array}$ & $\begin{array}{l}\text { O } 2015 \mathrm{~S} \text {. Karger AG, Basel } \\
\text { www.karger.com/cpb }\end{array}$ \\
\hline
\end{tabular}

and healthy controls. As shown in Fig. 2, plasma levels of HULC and Linc00152 increased dramatically in HCC patients as well as in training phase. However, no significant difference of UCA1 level was observed between the two groups. Besides, the expression of HULC significantly correlated with tumor size and tumor capsular. Moreover, the expression of Linc00152 was significantly associated with differentiation grade, tumor size, TNM stage and tumor capsular. These findings suggested that HULC and Linc00152 might play an important role in tumor occurrence, tumor growth and tumor invasion. Apparently, higher levels of HULC and Linc00152 implicated a higher risk of tumor occurrence, growth and invasion of HCC. Accordingly, we hypothesized that these two lncRNAs might act as biomarkers in predicting the occurrence, growth, or metastasis in HCC. ROC curve analysis showed the evidence for great value of HULC and Linc00152 in predicting the diagnosis of HCC. Taken together, the results demonstrated that both of HULC and Linc00152 reached a well AUC and the combination of HULC, Linc00152 and AFP got the highest AUC in predicting oncogenesis, tumor growth, and tumor metastasis. As a result, HULC and Linc00152 can act as novel biomarkers in predicting the diagnosis of HCC, and combination of HULC, Linc00152 and AFP get the highest prediction value of HCC.

In summary, we firstly identify that plasma HULC and Linc00152 were up-regulated dramatically in HCC patients. Both HULC and Linc00152 plasma levels indicate a significant predication of tumor growth and metastasis of HCC, the combination of HULC, Linc00152 and AFP would achieve better diagnostic accuracy. Thus, plasma HULC and Linc00152 can serve as potential noninvasive biomarkers for predicting the diagnosis of HCC. Furthermore, HULC and Linc00152 may be applied as a potential target for HCC treatment. However, this research is limited by the small sample size. Further large-scale studies are needed to confirm these findings.

\section{Acknowledgements}

This work was supported by grants from the National Natural Science Foundation for Distinguished Young Scholars (81225017 to B.S.); The State Key Program of National Natural Science of China (81430062 to B.S.); National Basic Research Program of China (2012CB910800 to B.S.), The National Natural Science Foundation (81201528 to R.J.). This work also supported in part by the Priority Academic Program of Jiangsu Higher Education Institutions. B.S. is Yangtze River scholars Distinguished Professor.

\section{Disclosure Statement}

The authors declared no conflict of interest.

\section{References}

1 Jemal A, Bray F, Center MM, Ferlay J, Ward E, Forman D: Global cancer statistics. CA Cancer J Clin 2011;61:69-90.

2 Tian H, Huang P, Zhao Z, Tang W, Xia J: HIF-1alpha plays a role in the chemotactic migration of hepatocarcinoma cells through the modulation of CXCL6 expression. Cell Physiol Biochem 2014;34:15361546.

3 Jiang HL, Xu D, Yu H, Ma X, Lin GF, Ma DY, Jin JZ: DAX-1 inhibits hepatocellular carcinoma proliferation by inhibiting beta-catenin transcriptional activity. Cell Physiol Biochem 2014;34:734-742.

4 Lafaro KJ, Demirjian AN, Pawlik TM: Epidemiology of Hepatocellular Carcinoma. Surg Oncol Clin N Am 2015;24:1-17.

5 Zhang YS, Chu JH, Cui SX, Song ZY, Qu XJ: Des-gamma-carboxy prothrombin (DCP) as a potential autologous growth factor for the development of hepatocellular carcinoma. Cell Physiol Biochem 2014;34:903-915.

6 Plentz RR, Boozari B, Malek NP: [Guideline compliant diagnostics of hepatocellular carcinoma]. Radiologe 2014;54:660-663.

7 Zhou L, Liu J, Luo F: Serum tumor markers for detection of hepatocellular carcinoma. World J Gastroenterol 


\section{Cellular Physiology Cell Physiol Biochem 2015;37:687-696 \begin{tabular}{l|l|l}
\hline DOI: 10.1159/000430387 & C 2015 S. Karger AG, Basel
\end{tabular} and Biochemistry Published online: September 11, 2015

Li et al.: HULC and Linc00152 as Biomarker

2006;12:1175-1181.

8 Schwarzenbach H, Hoon DS, Pantel K: Cell-free nucleic acids as biomarkers in cancer patients. Nat Rev Cancer 2011;11:426-437.

9 Zhou J, Lu S, Yang S, Chen H, Shi H, Miao M, Jiao B: MicroRNA-127 post-transcriptionally downregulates Sept7 and suppresses cell growth in hepatocellular carcinoma cells. Cell Physiol Biochem 2014;33:15371546.

10 Sanchez Y, Huarte M: Long non-coding RNAs: challenges for diagnosis and therapies. Nucleic Acid Ther 2013;23:15-20.

11 Kumarswamy R, Bauters C, Volkmann I, Maury F, Fetisch J, Holzmann A, Lemesle G, de Groote P, Pinet F, Thum T: Circulating long noncoding RNA, LIPCAR, predicts survival in patients with heart failure. Circ Res 2014;114:1569-1575.

12 Lorenzen JM, Schauerte C, Kielstein JT, Hubner A, Martino F, Fiedler J, Gupta SK, Faulhaber-Walter R, Kumarswamy R, Hafer C, Haller H, Fliser D, Thum T: Circulating Long Noncoding RNA TapSAKI Is a Predictor of Mortality in Critically Ill Patients with Acute Kidney Injury. Clin Chem 2015;61:191-201

13 Zheng HT, Shi DB, Wang YW, Li XX, Xu Y, Tripathi P, Gu WL, Cai GX, Cai SJ: High expression of lncRNA MALAT1 suggests a biomarker of poor prognosis in colorectal cancer. Int J Clin Exp Pathol 2014;7:31743181.

14 Wang F, Ren S, Chen R, Lu J, Shi X, Zhu Y, Zhang W, Jing T, Zhang C, Shen J, Xu C, Wang H, Wang H, Wang Y, Liu B, Li Y, Fang Z, Guo F, Qiao M, Wu C, Wei Q Xu D, Shen D, Lu X, Gao X, Hou J, Sun Y: Development and prospective multicenter evaluation of the long noncoding RNA MALAT-1 as a diagnostic urinary biomarker for prostate cancer. Oncotarget 2014;5:11091-11102.

15 Zhang Z, Hao H, Zhang CJ, Yang XY, He Q Lin J: [Evaluation of novel gene UCA1 as a tumor biomarker for the detection of bladder cancer]. Zhonghua Yi Xue Za Zhi 2012;92:384-387.

16 Srivastava AK, Singh PK, Rath SK, Dalela D, Goel MM, Bhatt ML: Appraisal of diagnostic ability of UCA1 as a biomarker of carcinoma of the urinary bladder. Tumour Biol 2014;35:11435-11442.

17 Han Y, Liu Y, Gui Y, Cai Z: Long intergenic non-coding RNA TUG1 is overexpressed in urothelial carcinoma of the bladder. J Surg Oncol 2013;107:555-559.

18 Nissan A, Stojadinovic A, Mitrani-Rosenbaum S, Halle D, Grinbaum R, Roistacher M, Bochem A, Dayanc BE, Ritter G, Gomceli I, Bostanci EB, Akoglu M, Chen YT, Old LJ, Gure AO: Colon cancer associated transcript-1: a novel RNA expressed in malignant and pre-malignant human tissues. Int J Cancer 2012;130:1598-1606.

19 Pang Q, Ge J, Shao Y, Sun W, Song H, Xia T, Xiao B, Guo J: Increased expression of long intergenic non-coding RNA LINC00152 in gastric cancer and its clinical significance. Tumour Biol 2014;35:5441-5447.

20 Zhang X, Gejman R, Mahta A, Zhong Y, Rice KA, Zhou Y, Cheunsuchon P, Louis DN, Klibanski A: Maternally expressed gene 3, an imprinted noncoding RNA gene, is associated with meningioma pathogenesis and progression. Cancer Res 2010;70:2350-2358.

21 Panzitt K, Tschernatsch MM, Guelly C, Moustafa T, Stradner M, Strohmaier HM, Buck CR, Denk H, Schroeder R, Trauner M, Zatloukal K: Characterization of HULC, a novel gene with striking up-regulation in hepatocellular carcinoma, as noncoding RNA. Gastroenterology 2007;132:330-342.

22 Lai MC, Yang Z, Zhou L, Zhu QQ Xie HY, Zhang F, Wu LM, Chen LM, Zheng SS: Long non-coding RNA MALAT-1 overexpression predicts tumor recurrence of hepatocellular carcinoma after liver transplantation. Med Oncol 2012;29:1810-1816.

23 Tu ZQ, Li RJ, Mei JZ, Li XH: Down-regulation of long non-coding RNA GAS5 is associated with the prognosis of hepatocellular carcinoma. Int J Clin Exp Pathol 2014;7:4303-4309.

24 Zhao J, Greene CM, Gray SG, Lawless MW: Long noncoding RNAs in liver cancer: what we know in 2014. Expert Opin Ther Targets 2014;18:1207-1218.

25 Seo SI, Kim SS, Choi BY, Lee SH, Kim SJ, Park HW, Kim HS, Shin WG, Kim KH, Lee JH, Kim HY, Jang MK: Clinical significance of elevated serum alpha-fetoprotein (AFP) level in acute viral hepatitis A (AHA). Hepatogastroenterology 2013;60:1592-1596.

26 Wong CR, Garcia RT, Trinh HN, Lam KD, Ha NB, Nguyen HA, Nguyen KK, Levitt BS, Nguyen MH: Adherence to screening for hepatocellular carcinoma among patients with cirrhosis or chronic hepatitis B in a community setting. Dig Dis Sci 2009;54:2712-2721.

27 Li H, Li Y, Liu D, Sun H, Liu J: miR-224 is critical for celastrol-induced inhibition of migration and invasion of hepatocellular carcinoma cells. Cell Physiol Biochem 2013;32:448-458.

28 Yang G, Lu X, Yuan L: LncRNA: a link between RNA and cancer. Biochim Biophys Acta 2014;1839:10971109. 